\title{
Endovenous cyanoacrylate ablation for chronic venous insufficiency and varicose veins among Asians
}

Sally SJ Chan, ${ }^{1}{ }_{M R C S}$, Yiu Che $\underline{\text { Chan }},{ }^{2}{ }_{F R C S}\left(\right.$ Gen) , Stewart R Walsh, ${ }^{3}$ FRCS (Gen), Tze Tec Chong, ${ }^{4,5}{ }_{F A C S}$, Edward TC Choke, ${ }^{6}{ }_{F R C S(G e n)}$, Alok Tiwari, ${ }^{7}$ FRCSEd, Tjun Yip Tang, ${ }^{4,5}{ }_{F R C S(G e n)}$

\begin{abstract}
Introduction: Endovenous cyanoacrylate glue (CAG) ablation for the treatment of chronic venous insufficiency (CVI) and varicose veins has shown non-inferior outcomes with an excellent safety profile, high patient satisfaction rate, and excellent efficacy when compared to the gold standard of endothermal ablation. A review of the current literature for CAG use in CVI showed that most studies and longer-term data are from Caucasian-based populations, which are subject to different anatomical venous variations and socio-economical contexts. This review aimed to gather the current evidence for CAG use in Asian CVI patients.

Methods: Asian studies for the use of CAG in CVI were included in this review. Successful ablation rates, quality of life improvement and novel complications such as glue hypersensitivity reactions are described, along with anatomical descriptions of superficial venous anatomy in study patients. Use of CAG in Singapore and Asia was addressed.

Results: CAG has been gaining traction as an option for CVI treatment in Asians. In Singapore, it has been adopted with comparable low complication rates and significant improvement of quality of life after treatment. As we increase our understanding of the variations in venous anatomy in the Asian population, new techniques such as retrograde deployment of the device and use of CAG ablation for venous leg ulcers have been developed.

Conclusion: Further robust evidence in terms of large randomised control trials along with costeffectiveness studies are needed to determine the true value of CAG ablation in the Asian setting.
\end{abstract}

Ann Acad Med Singap 2021;50:241-9

Keywords: Asian, chronic venous insufficiency, cyanoacrylate glue, endovenous, varicose veins

\section{MINIMALLY INVASIVE ENDOVENOUS TECHNIQUES}

In the past 2 decades, the management of chronic venous insufficiency (CVI) has been revolutionised by the introduction of minimally invasive endovenous techniques, which have replaced open surgical high tie and stripping as the treatment of choice. CVI is common in the Western population and is reported to affect 164 in 1,000 individuals. ${ }^{1}$ The prevalence of CVI in the Asian population is reportedly lower than that in the
non-Hispanic white population, ${ }^{2}$ but is expected to rise because of ageing and an increasing incidence of obesity. ${ }^{3}$ Some studies have shown that the Asian venous patient tends to present at a younger age with less severe symptoms, but these data may not be representative of the diverse Asian population. ${ }^{4,5}$

The guidelines of the Society for Vascular Surgery ${ }^{6}$ and the National Institute of Clinical Excellence (NICE) ${ }^{7}$ currently recommend the use of endothermal ablation techniques, specifically radiofrequency ablation (RFA)

\footnotetext{
${ }^{1}$ Department of General Surgery, Singapore General Hospital, Singapore

${ }^{2}$ Division of Vascular Surgery, Department of Surgery, The University of Hong Kong, Hong Kong

${ }^{3}$ Lambe Institute for Translational Discipline of Surgery, National University of Ireland, Ireland

${ }^{4}$ Department of Vascular Surgery, Singapore General Hospital, Singapore

${ }^{5}$ Duke-NUS Graduate Medical School, Singapore

${ }^{6}$ Department of General Surgery, Sengkang General Hospital, Singapore

${ }^{7}$ Department of Vascular Surgery, Queen Elizabeth Hospital, Birmingham, UK

Correspondence: Assoc Prof Tjun Yip Tang, Department of Vascular Surgery, Singapore General Hospital, Level 5, Academia Building, 20 College Road, Singapore 169856.

Email: tang.tjun.yip@singhealth.com.sg
} 


\section{CLINICAL IMPACT}

\section{What is New}

- Endovenous cyanoacrylate glue (CAG) ablation for chronic venous insufficiency and varicose veins has shown excellent safety profile, high patient satisfaction rate and excellent efficacy in the short term in Asian patients.

- New techniques for performing CAG ablation have been developed to adapt to variations in venous anatomy in Asian patients.

\section{Clinical Implications}

- CAG use is increasing in the Asian population, and has the potential to treat a wider group of chronic venous insufficiency patients, including those with venous leg ulcers.

and endovenous laser ablation (EVLA), as first-line treatment for truncal reflux, followed by ultrasoundguided foam sclerotherapy if RFA and EVLA are unavailable. The thermal energy used in RFA and EVLA to ablate the truncal vein can result, albeit rarely, in pain, skin burns, skin pigmentation, nerve damage, endothermal heat-induced thrombosis and arteriovenous fistula formation. ${ }^{8} 9$ Furthermore, due to the use of thermal energy, it is necessary to infiltrate the area around the whole length of the target truncal vein with tumescent anaesthesia through multiple injections, to minimise complications such as nerve injury and heat-related damage to surrounding structures (that act as a heat sink) and make the procedure more bearable for the patients, especially if performed under local anaesthesia. This in turn is also a source of intraprocedural and post-operative discomfort for patients and is known to cause ecchymosis and haematomas to some degree in more than $50 \%$ of treated patients. ${ }^{8}$

\section{NON-THERMAL, NON-TUMESCENT ABLATION TECHNIQUES}

Recently, non-thermal, non-tumescent ablation techniques (NTNTs) have been introduced to obviate the need for tumescence and the complications of thermal ablation. These NTNTs include mechanochemical ablation (MOCA) using the ClariVein device ${ }^{10}$ or sealing of veins by coaptation using cyanoacrylate glue (CAG). NICE has come up with guidelines for both MOCA and $\mathrm{CAG}$, highlighting both their safety profile and efficacy, while minimising perivenous tissue damage or pain. ${ }^{11,12}$ However, long-term data of both MOCA and CAG are not yet available, and thus they are not offered as the first line of treatment for CVI in the NICE guidelines algorithm. These NTNTs have led to a reduction in patient discomfort, haematoma formation and risks of nerve injury when compared with traditional thermal-based procedures, improving the patient's periprocedural experience further.

The current available evidence demonstrates high anatomical success rates for MOCA and CAG, with significant improvement in symptoms as demonstrated by improvement in quality of life as evidenced by the Venous Clinical Severity Score and Aberdeen Varicose Vein Questionnaire scores post-treatment. ${ }^{13} \mathrm{~A}$ recent network meta-analysis looking at 20 randomised controlled trials from 1996 to 2018 comparing different treatment options for saphenous reflux, including both MOCA and CAG, suggested that CAG had the highest probability of being ranked first in terms of anatomical success. CAG was also the most likely to reduce post-operative pain score from baseline, while having the lowest occurrence of adverse events. ${ }^{14}$

\section{Cyanoacrylate glue ablation}

The main chemical compound of CAG is N-butyl cyanoacrylate, which polymerises rapidly in the presence of ionic substances such as blood and tissue fluids. This polymerised form has excellent tensile strength and is the primary component of medical glues. Almeida et al. was the first group to study endovenous injections of CAG and demonstrated it to be safe and reliable for the treatment of CVI in human patients. ${ }^{15}$

There are 3 CAG delivery devices commercially available, designed specifically to treat superficial vein incompetence: VenaSeal, VariClose and VenaBlock systems. All 3 follow the same basic principles: N-butyl cyanoacrylate rapidly solidifies and creates an inflammatory reaction within the vein walls, and additional external compression over the vein opposes the endothelium together. The major difference between these devices is the viscosity and the polymerisation time of the glue, which affects the procedure, technique, duration and complication rates.

VenaSeal closure system (Medtronic, Dublin, Ireland) is the most studied CAG device for incompetent great saphenous vein (GSV) and small saphenous vein (SSV) ablation. It uses a rapidly polymerising, high-viscosity CAG to prevent potential embolisation into the deep venous system. ${ }^{16}$ Only 1 randomised controlled trial 
has been performed to date. The VenaSeal Sapheon Closure System Pivotal Study (VeClose), a multicentre prospective randomised controlled trial conducted in the US, evaluated VenaSeal versus RFA outcomes of 222 patients (108 received VenaSeal and 114 received RFA) over 3 years. ${ }^{17}$ The study found that VenaSeal was non-inferior to RFA in terms of treatment of incompetent GSV. These results were further validated by a multicentre prospective European trial. ${ }^{18}$

VariClose vein sealing system (Biolas Inc, Ankara, Turkey) is a newer device and is also utilised for both incompetent GSV and SSV ablation. It uses CAG with modifications to the chemical structure of N-butyl cyanoacrylate, resulting in lower viscosity and a faster polymerisation time compared with VenaSeal glue. There are no randomised controlled trials in the literature comparing VariClose to other techniques, but a systematic review of 1,000 limbs with VariClose suggests good efficacy with a 30-month occlusion rate of $94.1 \%$ and a high safety profile. ${ }^{19}$

VenaBlock system (Invamed, Ankara, Turkey) is the latest addition to the CAG armamentarium. It consists of a proprietary formula of N-butyl cyanoacrylate with dimethyl sulfoxide, which shortens the time for the initial polymerisation reaction to a mere 5 seconds. This dispensing system also has a guiding light at the tip of the catheter to visually guide the operator on the exact location requiring compression to ensure proper apposition within the fast polymerisation time. Two studies have been published in 2 independent patient populations in Turkey (total 1,111 patients), with promising reports of 12-month occlusion rates of $99.4 \%$ and reported rates of phlebitis in less than $2 \%$ of the population..$^{20,21}$

A review of the published literature revealed that the majority of device-related venous outcome data were from Caucasian-based cohorts, which may not extrapolate to other racial cohorts with variations in venous anatomy. This article evaluates the utility of CAG in Asian patients with CVI and varicose veins, and reviews the available literature.

\section{Anatomical variation in Asian populations}

An understanding of venous anatomical patterns and its variation in the Singapore population, in contrast to other populations, will help care providers determine if studies performed in other populations are applicable to its own patients.

Previous venous studies on the Asian sub-population from Western countries ${ }^{22,23}$ and small cohort reports from Thailand ${ }^{24}$ suggest that the characteristics of CVI in Asian patients differ from those found in Caucasian populations. Recently, Lee et al. studied the CVI population in Singapore and the US and found that Asians tend to have smaller truncal saphenous vein diameters and longer segments of reflux as compared with their Caucasian counterparts, and CVI tend to present at later stages in contrast to previous data on the Asian population. ${ }^{25}$ Interestingly, Asians were noted to have more advanced venous disease at presentation despite having veins of smaller diameters. This finding is in concordance with a study by Gibson et al. who demonstrated that GSV diameter is a poor surrogate marker for assessing the effect of varicose veins on a patient's quality of life. ${ }^{26}$

Anatomical difference also has its implications on the management of patients with CVI. Based on the Caucasian literature, CAG is indicated for patients with GSV diameters $>5 \mathrm{~mm}$. However, applying these guidelines to an Asian population whose median GSV diameters were $2.9 \mathrm{~mm}$ compared with $5.7 \mathrm{~mm}$ in the Caucasian population ${ }^{25}$ would lead to a severe undertreatment of Asian patients with CVI. Given the smaller diameter of their veins, it is likely that smaller aliquots of CAG are required to seal the vein than what is quoted in protocols and instructions for use designed for Caucasians.

Another significant finding by Lee et al. was that Asians had significantly longer lengths of venous reflux with a higher percentage of patients with reflux down to the ankle. This finding makes NTNTs even more suitable than thermal endovenous ablation for treatment of disease below the knee, with a significantly lower risk of saphenous nerve injury and skin damage.

The GSV exits the fascial envelope early in a larger proportion of Asians than in the Caucasian population, ${ }^{25}$ which invariably means that the Asian population has veins that lie more superficially and closer to the skin surface (termed N3 veins). This is in tandem with the lower body mass index noted in Asian populations than in Caucasian populations. ${ }^{27}$ This variation may result in a higher percentage of patients with thermal skin injury when using thermal endovascular ablation techniques such as RFA. Epifascial veins are also often tortuous and technically challenging to navigate endovenously and may lend themselves better to occlusion using CAG than thermal techniques. ${ }^{28}$

\section{Asian experience with CAG}

The first published VenaSeal outcome data from Asia were from Hong Kong in 2017, and focused on patients with bilateral varicose veins (predominantly 
$\mathrm{C} 2$ and $\mathrm{C} 3$ disease) because of cost concerns. ${ }^{29}$ Since then, other Asian countries, such as South Korea and Singapore, have also published their own experiences regarding VenaSeal use in their population. VenaSeal is the most commonly used and widely studied form of CAG in Asia. Two other CAG devices from Turkey (VariClose and VenaBlock) have recently entered the Singapore market. They have only been available at the institution of our last author (TYT), and local data on these systems have yet to be published. However, the experience has been satisfactory to date with fast procedural times and glue polymerisation rate.

CAG is indicated as treatment for patients with reflux of the saphenous trunks. In the initial reports from Western literature, the use of CAG was mostly limited to the treatment of GSV alone. However, its use was subsequently expanded to patients with reflux in the anterior accessory saphenous vein and $\mathrm{SSV}^{30}$ as well as incompetent perforators. These groups of patients have been included in the studies performed in the Asian population. ${ }^{31,32}$

In the Western literature, the 36-month occlusion rate of GSV has been quoted to be $92.9-94.7 \%{ }^{18,33,34}$ No 36-month data have been published for the Asian population, but a pioneer study in Hong Kong by Chan et al. (55 patients, 108 GSVs) reported a 12-month GSV occlusion rate of only $75.7 \% .{ }^{35} \mathrm{~A}$ further analysis of the results showed that the low rates could be related to patient selection and the instructions for use technique used, proving that GSV diameters of $>6.6 \mathrm{~mm}$ were at a higher risk of proximal recanalisation on follow-up. ${ }^{35}$ The closure rate of GSVs $<6.6 \mathrm{~mm}$ in diameter was $90 \%$ at 12 months post-operatively, but this value dropped to $58.6 \%$ for GSVs with diameters of $>6.6 \mathrm{~mm}$.

A Singapore study leveraged on this knowledge and described a double-dosing technique ${ }^{36}$ to improve truncal vein sealing rates. The investigators advocated delivering a double dose of $0.2 \mathrm{~mL}$ instead of the usual $0.1 \mathrm{~mL}$ at the saphenofemoral junction after the initial pullback of the delivery catheter. Where the GSV was focally dilated $(>6 \mathrm{~mm})$ and at the level where significant branches joined the truncal vein and where incompetent perforators were located, double dosing of CAG was performed, along with gentle external massage using the ultrasound probe to deliberately allow CAG dispersion into incompetent venous reservoirs to cause occlusion. Double-dosing was associated with a small risk of saphenofemoral junction occlusion as a result of the CAG creeping proximally into the saphenofemoral junction, but the patients were noted to be asymptomatic and were managed conservatively. A higher frequency of phlebitis was also noted in the double-dosing CAG group, which could possibly be attributed to the larger amount of CAG delivered focally. Subsequently, a study by the same investigators in 2019 (77 patients, 88 GSVs) reported a 12 -month occlusion rate of $91.5 \%,{ }^{31}$ which is comparable to results in the Western literature. These results were reproduced in a South Korean population by Park et al. (33 patients, 47 GSVs), where there was an initial outcome of $100 \%$ occlusion rate at 3 months, using additional CAG doses for regions with larger diameters, communicating veins or perforating veins. ${ }^{32}$ The migration of CAG proximally past the saphenofemoral junction into deep venous system runs the risk of developing deep vein thrombosis and pulmonary embolism. Conversely, if the CAG is deposited more distally than intended, treatment may be suboptimal with possibly increased risk of recurrence. Proximal CAG migration is subject to individual operator variability when the ultrasound compression is applied during the initial CAG deposition, and further studies have been carried out to investigate the factors that determine CAG migration at the saphenofemoral junction. Park and Kim initially reported an inverse relationship between GSV diameter and remnant stump, ${ }^{37}$ but a multivariate analysis by Lee et al., which employed the double-dosing technique for larger GSV veins (diameter $>6 \mathrm{~mm}$ ), subsequently showed that the maximum diameter of proximal GSV was predictive of shorter stump lengths post-procedure. ${ }^{38}$ The discrepancy in results is attributed to differences in methodology, with the volume of CAG delivered being a confounding factor, and further multicentre studies with standardised methodologies are required to evaluate this.

\section{Phlebitis and hypersensitivity reaction with CAG}

While CAG is overall safe and effective with the lowest odds of adverse events compared to other superficial venous therapies for CVI (including RFA, foam sclerotherapy, EVLA and MOCA), ${ }^{14}$ a hypersensitivity reaction to N-butyl cyanoacrylate is a risk that is unique to CAG treatment. This phenomenon has also been described as a "phlebitis-like abnormal reaction", characterised as a painless, itchy, erythematous cutaneous/dermal reaction distributed over the target vein where CAG is delivered into. ${ }^{39}$ Studies suggest that the phenomenon is a histotoxic inflammatory reaction, ${ }^{40}$ and more likely to be a foreign body or allergic reaction to CAG rather than venous phlebitis, which characteristically has the symptoms of pain, tenderness and swelling over the affected veins. 
Histological findings of the explanted vein in patients with more severe hypersensitivity reaction revealed lymphocytic follicles and giant cells typifying a chronic foreign body reaction, similar to findings of a vein explanted in healthy asymptomatic volunteers 5 years after CAGimplantation in GSV. ${ }^{41}$ Some authors have hypothesised that phlebitis-like abnormal reaction is likely due to a type IV hypersensitivity reaction, a delayed immune cell response mediated by $\mathrm{T}$ cells, noting that it occurred in both limbs of patients who have had undergone bilateral treatment, rather than being localised to a single limb. ${ }^{42}$ There is notably a delay between treatment and symptoms which usually occur 1-2 weeks after allergen exposure, in keeping with the pathophysiology of type IV hypersensitivity reaction. The reaction is frequently self-limiting and usually resolves with a short course of oral non-steroidal antiinflammatory agents and/or anti-histamines and/or steroids. More serious reactions have been reported, such as the development of multiple "painless large pustules" with surrounding erythema around the targeted veins post-treatment, with subsequent progression to the eruption of these pustules and extrusion of white CAG casts from each wound. ${ }^{43}$ Extra precautions are taken to minimise the contact of $\mathrm{CAG}$ with dermis and subcutaneous tissue, in which the retained polymer can serve as a nidus for infection or localised foreign body reaction, potentially requiring excision. This phenomenon can manifest as puncture site infections, postulated to be due to secondary gluenextravasation during sheath removal, and are observed to happen more frequently in patients undergoing treatment with $\mathrm{CAG}$ compared with other endovascular forms of treatment. ${ }^{44}$

It is unclear what the true rate of hypersensitivity reaction is among the different populations. Earlier studies, such as VeClose and the European Sapheon Closure System Observational Prospective Study (eSCOPE), have lumped this phenomenon with all occurrences of post-treatment phlebitis with reported rates of $18.5 \%{ }^{33}$ and $11.4 \%,{ }^{18}$ respectively. A study by Gibson et al. performed in the US documented that $6 \%$ of their patients experienced hypersensitivity reaction, but did not find any differences in the frequency of the condition by race in their patient population. However, it is important to note that there were only 23 non-white patients out of a total of 286 patients in the cohort, ${ }^{41}$ resulting in sampling bias. In contrast, Park reported a rate of $25.4 \%$ for 271 veins treated in a South Korean population, ${ }^{32}$ while Tang et al. reported that it affected $18 \%$ of patients undergoing CAG treatment in a multicentre Asian study conducted in Singapore. ${ }^{31}$ The increased rate of hypersensitivity reaction in the Asian population may be due to differences in genetics and environmental conditions. ${ }^{45}$ Anatomically, suprafascial GSVs are more common in the Asian population than in the Western population, and have a significant correlation with higher hypersensitivity reaction rates as the suprafascial location is closer to the skin and hence may have more pronounced signs and symptoms. The study by Park et al. showed that hypersensitivity reaction occurrences were significantly higher in the GSV group than in the SSV group, and even more so in cases with a suprafascial GSV length of $>10 \mathrm{~cm}^{42}$

Current studies advocate the exclusion of patients with CAG allergies when considering CAG ablation for CVI treatment, and prophylactic doses of oral nonsteroidal anti-inflammatory agents or steroids may be useful for minimising the occurrence of hypersensitivity reaction events. The last author (TYT) has stopped offering CAG ablation to patients with multiple drug allergies as hypersensitivity reaction has been observed to be higher in these patients. ${ }^{31}$ Another problem patients face following CAG ablation is the pulling of the fibrosed truncal vein under the skin when they bend and extend their knee. This problem is more pronounced when the GSV lies close to the surface of the skin, especially in the distal thigh and proximal calf area. ${ }^{31}$ We advocate not treating these superficial axial veins with glue. Further studies are required to define patient or procedural risk factors for hypersensitivity reaction, for better patient selection and techniques to decrease the frequency.

\section{Satisfaction rates in Asian CAG ablation patients}

A review of the Venous Clinical Severity Score and Aberdeen Varicose Vein Questionnaire scores in Asian populations showed marked improvement postprocedure, consistent with the experience in Western populations (Table 1). The baseline Venous Clinical Severity Scores were higher in the Asian population, which translated to a greater improvement in the scores post-CAG treatment.

The pain scores on post-operative day 1 in the Asian population remained low at 3 or less on the Visual Analogue Scale, and these are comparative to those in the Western population. Patient satisfaction scores remained high across both populations, with $82.6 \%$ and $87.0 \%$ of patients in the VeClose study ${ }^{30}$ and Singapore cohor $^{46}$ respectively, with the majority of patients stating that they would definitely choose CAG ablation again if given the choice. 
Table 1. Venous Clinical Severity Score and Aberdeen Varicose Vein Questionnaire scores in Western and Asian populations

\begin{tabular}{|c|c|c|c|c|}
\hline \multirow[t]{2}{*}{ Author/Study } & \multicolumn{2}{|c|}{ Mean VCSS score } & \multicolumn{2}{|c|}{ Mean AVVQ score } \\
\hline & Baseline & $\begin{array}{c}\text { Follow-up } \\
\text { (months post-operatively) }\end{array}$ & Baseline & $\begin{array}{c}\text { Follow-up } \\
\text { (months post-operatively) }\end{array}$ \\
\hline VeClose study, USA ${ }^{\mathrm{a}}$ & 5.5 & $<2.0$ (12 months) & 18.9 & 9 (12 months) \\
\hline eSCOPE study, Europe ${ }^{\mathrm{b}}$ & 4.3 & 1.1 (12 months) & 16.4 & 6.7 (12 months) \\
\hline Chan et al., Hong Kong ${ }^{c}$ & 6.9 & 1.7 (12 months) & 23.7 & 4.1 (12 months) \\
\hline Tang et al., Singapore ${ }^{d}$ & 6.6 & 3.5 (3 months) & 17.1 & 4.8 (3 months) \\
\hline Park, South Korea ${ }^{e}$ & 4.2 & 1.2 (1 month) & Not reported & Not reported \\
\hline
\end{tabular}

AVVQ: Aberdeen Varicose Vein Questionnaire; VCSS: Venous Clinical Severity Score

${ }^{a}$ Morrison N, Gibson K, McEnroe S, et al. Randomized trial comparing cyanoacrylate embolization and radiofrequency ablation for incompetent great saphenous veins (VeClose). J Vasc Surg 2015;61:985-94.

${ }^{\mathrm{b}}$ Proebstle TM, Alm J, Dimitri S, et al. The European multicenter cohort study on cyanoacrylate embolization of refluxing great saphenous veins. J Vasc Surg Venous Lymphat Disord 2015;3:2-7.

${ }^{\mathrm{c}}$ Chan YC, Law Y, Cheung GC, et al. Cyanoacrylate glue used to treat great saphenous reflux: Measures of outcome. Phlebology 2017;32:99-106.

d Tang TY, Rathnaweera HP, Kam JW, et al. Endovenous cyanoacrylate glue to treat varicose veins and chronic venous insufficiencyexperience gained from our first $100+$ truncal venous ablations in a multi-ethnic Asian population using the Medtronic VenaSeal ${ }^{\mathrm{TM}}$ Closure System. Phlebology 2019;34:543-51.

${ }^{e}$ Park I. Initial outcomes of cyanoacrylate closure, VenaSeal system, for the treatment of the incompetent great and small saphenous veins. Vasc Endovascular Surg 2017;51:545-9.

\section{CAG use in Singapore}

In Singapore, our main experience with CAG devices is with VenaSeal, which was first available in Singapore in January 2016. It has been approved by the Health Sciences Authority and is used by both the public and private healthcare sectors for CVI treatment. The other 2 CAG devices (VariClose and VenaBlock) have also been approved by the Health Sciences Authority and are generally cheaper than VenaSeal, but no published outcomes in Asia exist as yet. NTNTs are now offered frequently as the treatment of choice for patients with CVI in Singapore, gaining traction over more conventional therapies such as RFA and foam sclerotherapy. Many patients choose CAG for treatment of their CVI, as doing so would eliminate the need for wearing compression stockings post-procedure. ${ }^{45}$ Conservative treatment with compression stockings is associated with low compliance rates, ${ }^{47}$ and in countries with a perennial hot and humid climate such as Singapore, compliance may be even lower.

Many techniques have been pioneered to overcome the anatomical variations seen in the Singapore population. Epifascial veins found among the local population are often tortuous and of a small diameter, and a double puncture technique ${ }^{48}$ has been employed to overcome this anatomical challenge. Retrograde puncture techniques ${ }^{49}$ with $\mathrm{CAG}$ have also been described from the proximal calf or thigh region to ensure that the distal portions of the GSV or SSV are adequately sealed. All these adjunct techniques, in addition to the instructions for use from the manufacturers, have enhanced the use of CAGs for the Asian population. NTNTs have also allowed the treatment of both GSV and SSV simultaneously with little risk to the saphenous nerve and sural nerve, respectively. This is particularly useful in a population with a higher prevalence of concomitant GSV and SSV reflux $(83.0 \%$ in the Singapore cohort versus $32.7 \%$ in the US cohort, $P<0.01){ }^{25}$ Post-procedurally, patients return to normal daily activities at a mean of 5 days (interquartile range 3-7 days), and to work after 10 days (interquartile range $7-14$ days). ${ }^{31}$

The techniques and benefits of NTNTs have rendered CAG relevant to the treatment of CVI in patients with venous ulcers. A preliminary study in Singapore has shown that concomitant $\mathrm{CAG}$ therapy with regular 4-layer compression bandaging decreased the time required for venous leg ulcers to heal and resulted in increased patient satisfaction rate with a significant decrease in Venous Clinical Severity Score and pain scores. ${ }^{50}$ Various puncture techniques were employed, including the double puncture technique (Fig. 1), retrograde puncture technique at the thigh (Fig. 2) and knee (Fig. 3), in addition to the conventional antegrade puncture technique at the ankle (Fig. 4). These techniques allowed the delivery of CAG directly to the vein below the ulcer to ensure obliteration of the underlying venous plexus, without the risks of skin burns or nerve injuries from endothermal ablation. The use of VenaSeal in Singapore may be precluded by its 


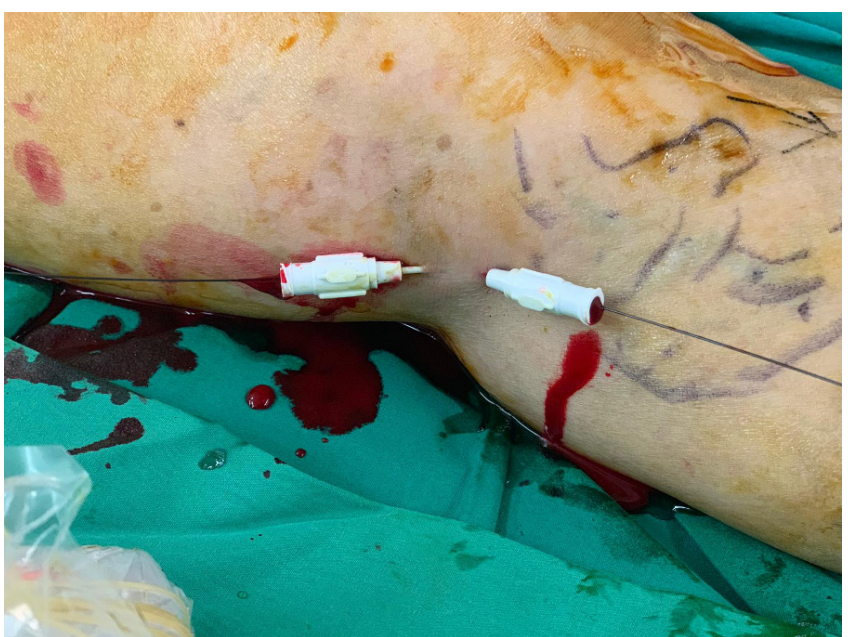

Fig. 1. Bidirectional puncture technique with 2 punctures at the knee in both antegrade and retrograde directions.

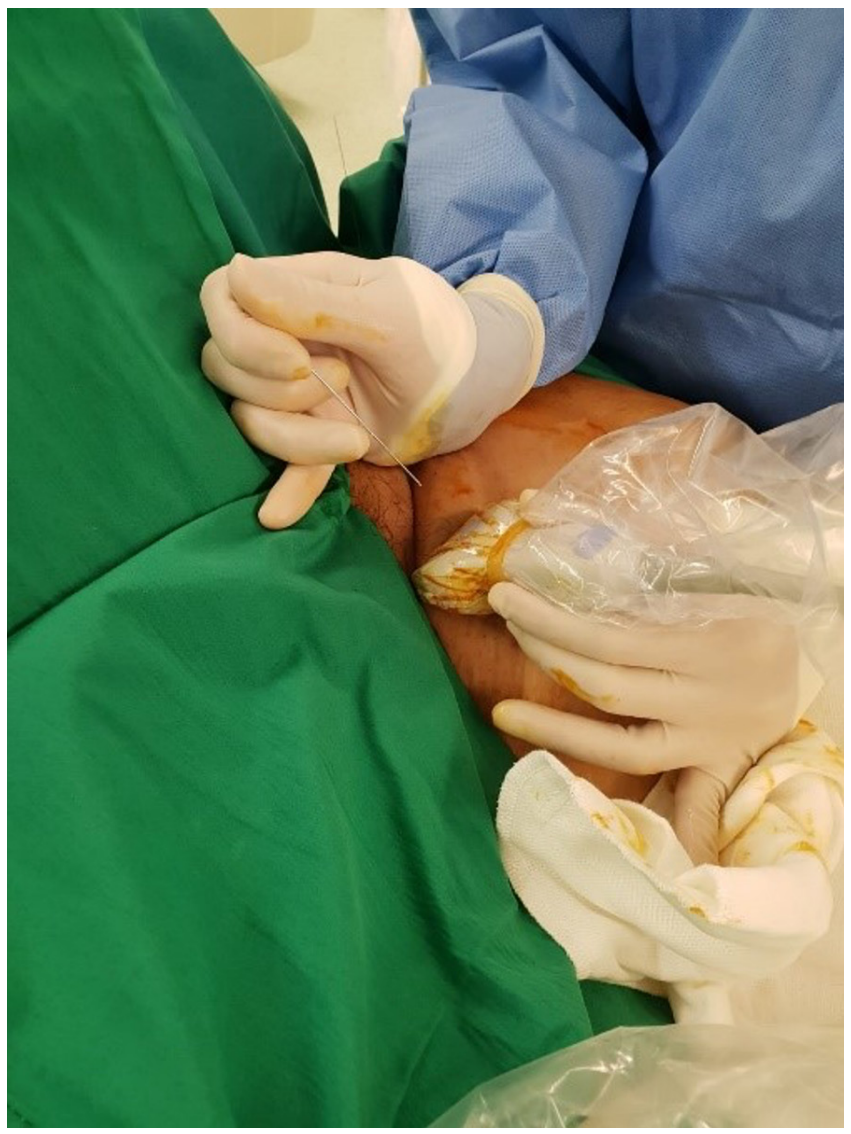

Fig. 2. Retrograde puncture technique with puncture site at the thigh, just below the saphenofemoral junction.

higher cost. Additionally, the cost has to be borne in cash by patients unless it is covered by the patient's private insurance. Cost is a barrier for CAG use in patients, especially patients who require government healthcare subsidy.

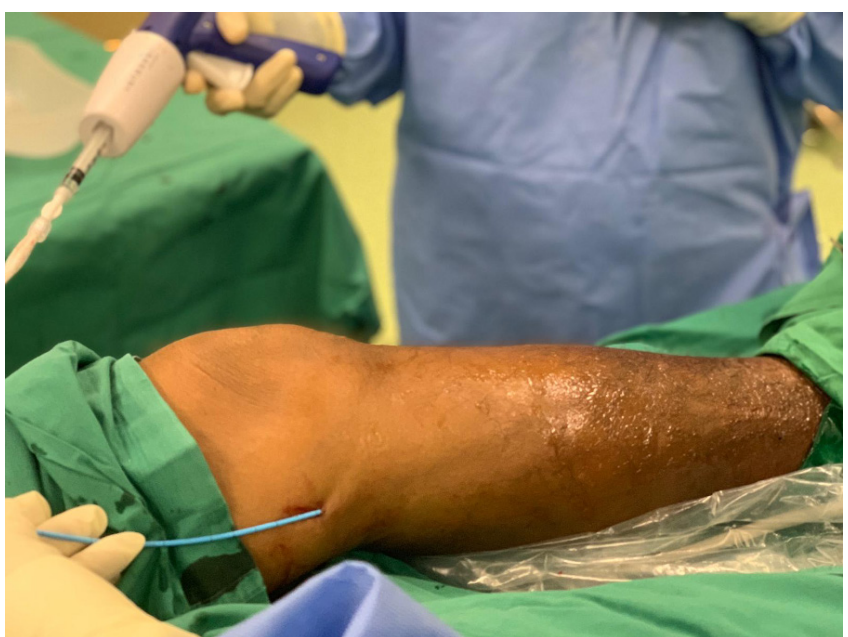

Fig. 3. Retrograde puncture technique with puncture site at the knee for reflux below the knee.

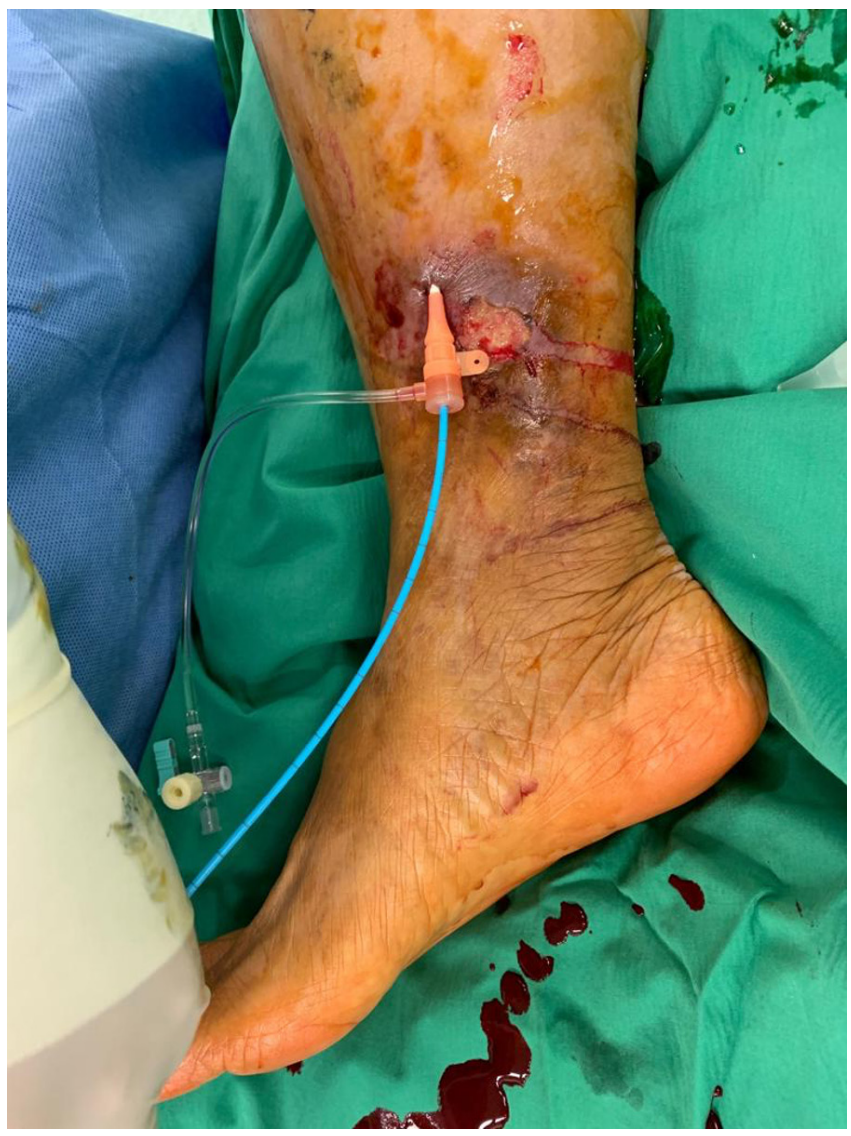

Fig. 4. Antegrade puncture technique with the puncture site at the ankle, next to the ulcer site.

\section{CONCLUSION}

CAG has been proven to be non-inferior to conventional methods for treating CVI, and is increasingly being used in both Western and Asian populations. As we increase our understanding of anatomical variations in 
the Asian population, new techniques of CAG beyond the instructions for use have been developed to complement this knowledge. These techniques will increase the range of treatment options for patients with CVI, and provides a potential for CAG to treat a wider group of CVI patients, including those with venous leg ulcers. CAG treatment costs are high. Further robust evidence in terms of large randomised controlled trials along with cost-effectiveness studies is needed to determine the value of CAG in the Asian setting.

\section{Disclosure}

The authors received no financial funding for the research, authorship or publication of this article. The last author (TYT) received investigator-initiated grants from Medtronic Singapore and Medtronic US for a separate VenaSeal-related study, which has completed patient recruitment and a 3-month result paper has been published (ASVS trial NCT03893201).

\section{REFERENCES}

1. Cesarone MR, Belcaro G, Nicolaides AN, et al. 'Real' epidemiology of varicose veins and chronic venous diseases: The San Valentino Vascular Screening Project. Angiology 2002;53:119-30.

2. Criqui MH, Jamosmos M, Fronek A, et al. Chronic venous disease in an ethnically diverse population: The San Diego Population Study. Am J Epidemiol 2003;158:448-56.

3. Youn YJ, Lee J. Chronic venous insufficiency and varicose veins of the lower extremities. Korean J Intern Med 2019;34:269-83.

4. Kanchanabat B, Wongmahisorn Y, Stapanavatr W, et al. Clinical presentation and patterns of venous reflux in Thai patients with chronic venous insufficiency (CVI). Eur J Vasc Endovasc Surg 2010;40:399-402.

5. Rabe E, Guex JJ, Puskas A, et al. Epidemiology of chronic venous disorders in geographically diverse populations: Results from the Vein Consult Program. Int Angiol 2012;31:105-15.

6. Gloviczki P, Comerota AJ, Dalsing MC, et al. The care of patients with varicose veins and associated chronic venous diseases: Clinical practice guidelines of the Society for Vascular Surgery and the American Venous Forum. J Vasc Surg 2011;53(5 Suppl):2S-48S.

7. National Institute for Health and Care Excellence. Varicose veins: Diagnosis and Management, 24 July 2013. Available at: https://www. nice.org.uk/guidance/cg168/resources/varicose-veins-diagnosis- andmanagement-pdf-35109698485957. Accessed on 25 May 2020.

8. Dexter D, Kabnick L, Berland T, et al. Complications of endovenous lasers. Phlebology 2012;27 Suppl 1:40-5.

9. Elias S, Raines JK. Mechanochemical tumescentless endovenous ablation: Final results of the initial clinical trial. Phlebology 2012;27:67-72.

10. Khor SN, Lei J, Kam JW, et al. ClariVein ${ }^{\mathrm{TM}}$ —One year results of mechano-chemical ablation for varicose veins in a multi-ethnic Asian population from Singapore. Phlebology 2018;33:687-94.

11. National Institute for Health and Care Excellence. Endovenous mechanochemical ablation for varicose veins: Interventional procedures guidance [IPG557], 25 May 2016. Available at: https://www. nice.org.uk/guidance/ipg557. Accessed on 26 May 2020.

12. National Institute for Health and Care Excellence. Cyanoacrylate glue occlusion for varicose veins: Interventional procedures guidance [IPG670], 4 March 2020. Available at https://www.nice.org. uk/guidance/ipg670. Accessed on 26 May 2020.

13. Vos CG, Ünlü Ç, Bosma J, et al. A systematic review and metaanalysis of two novel techniques of nonthermal endovenous ablation of the great saphenous vein. J Vasc Surg Venous Lymphat Disord 2017;5:880-96.

14. Kolluri R, Chung J, Kim S, et al. Network meta-analysis to compare VenaSeal with other superficial venous therapies for chronic venous insufficiency. J Vasc Surg Venous Lymphat Disord 2020;8:472-81.e3.

15. Almeida JI, Javier JJ, Mackay E, et al. First human use of cyanoacrylate adhesive for treatment of saphenous vein incompetence. J Vasc Surg Venous Lymphat Disord 2013;1:174-80.

16. Lam YL, De Maeseneer M, Lawson J, et al. Expert review on the VenaSeal ${ }^{\circledR}$ system for endovenous cyano-acrylate adhesive ablation of incompetent saphenous trunks in patients with varicose veins. Expert Rev Med Devices 2017;14:755-62.

17. Morrison N, Gibson K, McEnroe S, et al. Randomized trial comparing cyanoacrylate embolization and radiofrequency ablation for incompetent great saphenous veins (VeClose). J Vasc Surg 2015;61:985-94

18. Proebstle TM, Alm J, Dimitri S, et al. The European multicenter cohort study on cyanoacrylate embolization of refluxing great saphenous veins. J Vasc Surg Venous Lymphat Disord 2015;3:2-7.

19. Bissacco D, Stegher S, Calliari FM, et al. Saphenous vein ablation with a new cyanoacrylate glue device: A systematic review on 1000 cases. Minim Invasive Ther Allied Technol 2019;28:6-14.

20. Sarac A. Two-year follow-up of a n-butyl-2-cyanoacrylate glue ablation for the treatment of saphenous vein insufficiency with a novel application catheter with guiding light. Vascular 2019;27:352-8.

21. Yavuz T, Acar AN, Aydin H, et al. A retrospective study of a new n-butyl-2-cyanoacrylate glue ablation catheter incorporated with application guiding light for the treatment of venous insufficiency: Twelve-month results. Vascular 2018;26:547-55.

22. Hobbs SD, Sam R, Rehman A, et al. The utilisation of superficial venous surgery for chronic venous insufficiency by the U.K. Asian population. Eur J Vasc Endovasc Surg 2003;26:322-4.

23. Sam RC, Hobbs SD, Darvall KA, et al. Chronic venous disease in a cohort of healthy UK Asian men. Eur J Vasc Endovasc Surg 2007;34:92-6.

24. Kanchanabat B, Stapanavatr W. Venous ultrasonography findings and clinical correlations in 104 Thai patients with chronic venous insufficiency of the legs. Singapore Med J 2018;59:155-8.

25. Lee Q, Gibson K, Chan SL, et al. A comparison between Caucasian and Asian superficial venous anatomy and reflux patterns-Implications for potential precision endovenous ablation therapy. Phlebology 2020;35:39-45.

26. Gibson K, Meissner M, Wright D. Great saphenous vein diameter does not correlate with worsening quality of life scores in patients with great saphenous vein incompetence. J Vasc Surg 2012; $56: 1634-41$

27. Wulan SN, Westerterp KR, Plasqui G. Ethnic differences in body composition and the associated metabolic profile: A comparative study between Asians and Caucasians. Maturitas 2010;65:315-9.

28. Tang TY. Endovenous cyanoacrylate super-glue to treat varicose veins: How I perform VenaSeal ${ }^{\mathrm{TM}}$ ablation-Tips \& tricks gained 
from ablating over 300 truncal saphenous veins. Ann Vasc Med Surg 2018;1:1006.

29. Chan YC, Law Y, Cheung GC, et al. Cyanoacrylate glue used to treat great saphenous reflux: Measures of outcome. Phlebology 2017; 32:99-106.

30. Gibson K, Morrison N, Kolluri R, et al. Twenty-four month results from a randomized trial of cyanoacrylate closure versus radiofrequency ablation for the treatment of incompetent great saphenous veins. J Vasc Surg Venous Lymphat Disord 2018;6:606-13.

31. Tang TY, Rathnaweera HP, Kam JW, et al. Endovenous cyanoacrylate glue to treat varicose veins and chronic venous insufficiency - experience gained from our first 100+ truncal venous ablations in a multi-ethnic Asian population using the Medtronic VenaSeal $^{\text {TM }}$ Closure System. Phlebology 2019;34:543-51.

32. Park I. Initial outcomes of cyanoacrylate closure, VenaSeal system, for the treatment of the incompetent great and small saphenous veins. Vasc Endovascular Surg 2017;51:545-9.

33. Morrison N, Kolluri R, Vasquez M, et al. Comparison of cyanoacrylate closure and radiofrequency ablation for the treatment of incompetent great saphenous veins: 36-Month outcomes of the VeClose randomized controlled trial. Phlebology 2019;34:380-90.

34. Almeida JI, Javier JJ, Mackay EG, et al. Thirty-sixth-month follow-up of first-in-human use of cyanoacrylate adhesive for treatment of saphenous vein incompetence. J Vasc Surg Venous Lymphat Disord 2017;5:658-66

35. Chan YC, Law Y, Cheung GC, et al. Predictors of recanalization for incompetent great saphenous veins treated with cyanoacrylate glue. J Vasc Interv Radiol 2017;28:665-71

36. Tang TY, Rathnaweera HP, Choke E, et al. Institution of a double-dosing protocol using cyanoacrylate glue for larger diameter refluxing superficial truncal veins. Dermatol Surg 2019;45:1426-9.

37. Park I, Kim D. Correlation between the immediate remnant stump length and vein diameter after cyanoacrylate closure using the VenaSeal system during treatment of an incompetent great saphenous vein. Vasc Endovascular Surg 2020;54:47-50.

38. Lee TS, Chan SL, Rathnaweera HP, et al. Predictive factors for changes in remnant stump length during and after the treatment of incompetent truncal saphenous veins using cyanoacrylate. Phlebology 2020;35:605-13

39. Tang TY, Tiwari A. The VenaSeal ${ }^{\mathrm{TM}}$ abnormal red skin reaction: Looks like but is not phlebitis! Eur J Vasc Endovasc Surg 2018;55:841.
40. Hirsch T. Non-thermal endovenous treatment: acrylate adhesion of varicose saphenous veins. Phlebologie 2017;46:143-7.

41. Gibson K, Minjarez R, Rinehardt E, et al. Frequency and severity of hypersensitivity reactions in patients after VenaSealTM cyanoacrylate treatment of superficial venous insufficiency. Phlebology 2020;35:337-44

42. Park I, Jeong MH, Park CJ, et al. Clinical features and management of "phlebitis-like abnormal reaction" after cyanoacrylate closure for the treatment of incompetent saphenous veins. Ann Vasc Surg $2019 \cdot 55 \cdot 239-45$

43. Lew PS, Tan YK, Chong TT, Tang TY. VenasealTM cyanoacrylate glue rejection following endovenous ablation-Another new complication. Biomed J Sci Tech Res 2019;17:12993-4.

44. Sumarli SA, Lee QWS, Yap HY, et al. Exit site complications following cyanoacrylate glue endovenous ablation of incompetent truncal veins for chronic venous insufficiency. J Vasc Surg Cases Innov Tech 2020;6:500-4.

45. Alijotas-Reig J, Garcia-Gimenez V. Delayed immune-mediated adverse effects related to hyaluronic acid and acrylic hydrogel dermal fillers: Clinical findings, long-term follow-up and review of the literature. J Eur Acad Dermatol Venereol 2008;22:150-61.

46. Tang TY, Yap CJQ, Chan SL, et al. Early results of an Asian prospective multicentre VenaSeal real world postmarket evaluation to investigate the efficacy and safety of cyanoacrylate endovenous ablation for varicose veins. J Vasc Surg Venous Lymphat Disord 2021;9:335-45.e2

47. Raju S, Hollis K, Neglen P. Use of compression stockings in chronic venous disease: Patient compliance and efficacy. Ann Vasc Surg 2007;21:790-5

48. Tiwari A, Chong TT, Wang JCC, et al. Regarding "Double prepuncture as a valuable adjunctive technique for complex endovenous ablation". J Vasc Surg Venous Lymphat Disord 2018;6:420.

49. Chan SS, Tang TY, Chong TT, et al. Retrograde technique for great saphenous vein ablation using the VenaSeal ${ }^{\mathrm{TM}}$ closure system-Ideal approach for deep seated or small below the knee refluxing truncal veins. Phlebology 2020;35:102-9.

50. Chan SSJ, Yap CJQ, Tan SG, et al. The utility of endovenous cyanoacrylate glue ablation for incompetent saphenous veins in the setting of venous leg ulcers. J Vasc Surg Venous Lymphat Disord 2020;8:1041-8. 